\title{
Prevalência dos tumores cutâneos de equinos diagnosticados no Laboratório de Patologia Veterinária da Universidade Federal de Santa Maria, Rio Grande do Sul ${ }^{1}$
}

\author{
Tatiana M. Souza ${ }^{2}$, Juliana S. Brum ${ }^{3}$, Rafael A. Fighera ${ }^{4}$, Karin E. Brass ${ }^{4}$ e Claudio S.L. Barros ${ }^{4}$
}

\begin{abstract}
Souza T.M., Brum J.S., Fighera R.A., Brass K.E. \& Barros C.S.L. 2011. [Prevalence of equine skin tumors diagnosed at the Laboratory of Veterinary Pathology of the Federal University Santa Maria, Rio Grande do Sul, Brazil.] Prevalência dos tumores cutâneos de equinos diagnosticados no Laboratório de Patologia Veterinária da Universidade Federal de Santa Maria, Rio Grande do Sul. Pesquisa Veterinária Brasileira 31(5):379-382. Departamento de Patologia, Universidade Federal de Santa Maria, Camobi, Santa Maria, RS 97105-900, Brazil. E-mail: teitsouza@ yahoo.com.br

The aim of this study was to determine the prevalence of the equine skin tumors diagnosed at the Laboratory of Veterinary Pathology (LPV) of the Universidade Federal de Santa Maria (UFSM). Cases of equine skin biopsies diagnosed between January 1999 and December 2009 and filed at the LPV-UFSM were reviewed. In all cases the morphological diagnosis considered was the one written in the filed biopsy report. During the period studied 139 equine skin biopsies were submitted to the LPV-UFSM. Out of those, $108(77.6 \%)$ were skin tumors, neoplastic or otherwise and 31 cases were non-tumoral dermatosis. Fifty three of the 108 horses were females (49.1\%), 37 (34.2\% \%) were males and in 18 cases $(16.7 \%)$ the sex was not stated in the report. Sixty six affected horse $(61.1 \%)$ were purebreds, 13 $(12 \%)$ were crosses and in 29 instances $(26.9 \%)$ the breed was not stated in the report. The Crioulo was the most prevalent breed (44/108 [40.7\%]) and the remaining 22 purebreds horses were distribute among seven different breed. The most prevalent age categories affected in decreasing order were $1-5$ year-old (47/108 [43.5\%]) and 6-14 year-old (21/108 [19.5\%]); 12 horses $(11,1 \%)$ were 15 year-old or above .Only one horse was less than 1year-old. In 27 reports the age was not mentioned. More prevalent tumors included equine sarcoid (62/108 [57.4\%]); squamous cell carcinomas (11/108 [10.2\%]); pythiosis (9/108 [8.3\%]); granulation tissue ("proud flesh") (7/108 [6.5\%]) and eosinophilic granuloma (4/108 [3.8\%]). The remaining tumors accounted for approximately $14 \%$ of the cases.
\end{abstract}

INDEX TERMS: Diseases of horses, skin diseases, dermatology, dermatopathology.

RESUMO.- Este estudo teve como objetivo determinar a prevalência das dermatopatias tumorais de equinos diagnosticadas no Laboratório de Patologia Veterinária (LPV) da

\footnotetext{
${ }^{1}$ Recebido em 12 de novembro de 2010.

Aceito para publicação em 26 de novembro de 2010.

2 Pós-Doutorado, área de concentração em Patologia Veterinária, Laboratório de Patologia Veterinária, Departamento de Patologia, Centro de Ciências da Saúde, Universidade Federal de Santa Maria (UFSM), Camobi, Santa Maria, RS 97105-900, Brasil. *Autor para correspondência: teitsouza@yahoo.com.br

${ }^{3}$ Programa de Pós-Graduação em Medicina Veterinária, área de concentração em Patologia Veterinária, Centro de Ciências Rurais (CCR), UFSM, Santa Maria, RS 97105-900.

${ }^{4}$ Laboratório de Patologia Veterinária, Departamento de Patologia, Centro de Ciências da Saúde, UFSM, Santa Maria, RS.

${ }^{5}$ Departamento de Clínica de Grandes Animais, Hospital Veterinário, UFSM, Santa Maria, RS.
}

Universidade Federal de Santa Maria (UFSM), Rio Grande do Sul. Para isso, foram revisados os protocolos de biópsia de pele de equinos, arquivados no LPV-UFSM, entre janeiro de 1999 e dezembro de 2009. Em todos os casos, foram considerados os diagnósticos morfológicos que constavam nos protocolos. Durante esse período foram submetidas ao LPV-UFSM 139 amostras cutâneas de equinos. Dessas 139 amostras, 108 (77,6\%) eram de tumores cutâneos, neoplásicos ou não; os outros 31 casos consistiam de dermatoses não tumorais. Dos 108 equinos, $53(49,1 \%)$ eram fêmeas e $37(34,2 \% \%)$ eram machos, em $18(16,7 \%)$ casos não estava descrito no protocolo o sexo. Quanto à raça, $66(61,1 \%)$ eram de raça pura e 13 (12\%) não tinham raça definida; em $29(26,9 \%)$ protocolos não havia a descrição da raça. A raça mais prevalente foi a Crioula $(44 / 108[40,7 \%])$, o restante dos 22 equinos de raça pura pertencia a outras sete 
raças diferentes. Quanto à idade, as categorias mais prevalentes em ordem decrescente foram a de 1-5 anos (47/108 [43,5\%]) e a de 6-14 anos (21/108 [19,5\%]), cavalos com 15 anos ou mais representaram 11,1\% (12/108). Apenas um cavalo tinha menos de um ano de idade. Em 27 protocolos não constava a idade. Os tumores mais prevalentes incluíram: sarcoide $(62 / 108$ [57,4\%]); carcinoma de células escamosas (11/108 [10,2\%]); pitiose (9/108 [8,3\%]); tecido de granulação (7/108 [6,5\%]; e o granuloma eosinofílico (4/108 [3,8\%]). Os outros tumores contaram com aproximadamente $14 \%$ dos casos.

TERMOS DE INDEXAÇÃO: Doenças de equinos, doenças de pele, dermatologia, dermatopatologia.

\section{INTRODUÇÃO}

As lesões de pele estão entre as queixas clínicas mais frequentes em medicina equina e, embora não sejam um grande problema econômico, causam prejuízo estético. Depois do cão e do gato, o equino é a espécie doméstica mais comumente atendida devido a algum problema dermatológico (Scott \& Miller Jr 2003). Um estudo realizado entre 1962 e 1963 pela British Equine Veterinary Association demonstrou que $2 \%$ de todos os equinos encaminhados ao atendimento veterinário na Inglaterra apresentavam problemas de pele como queixa primária da consulta (Scott \& Miller Jr 2003). Outro estudo, realizado em 1989 pelos membros da American Association of Equine Practioners, revelou que as doenças de pele são o quarto problema mais comum em equinos dos Estados Unidos (TraubDargatz et al. 1991). Um estudo retrospectivo (1979-2000) realizado no College of Veterinary Medicine da Cornell University demonstrou que $4,1 \%$ de todos os equinos examinados na clínica de grandes animais tinham algum tipo de problema de pele (Scott 2007).

Dentre as dermatopatias, os tumores cutâneos são uma condição importante em equinos e, de acordo com alguns autores (Baker \& Leyland 1975), representam 50\% de todos os tumores que acometem essa espécie. Relatos descrevem que a prevalência de todos os tipos de neoplasmas em cavalos é superior a 3\% (Cotchin 1977) e que de todos os materiais de equinos recebidos em um serviço de diagnóstico histopatológico, $18 \%$ deles são neoplasmas (Kerr \& Alden 1974).

A prevalência das principais dermatopatias de equinos nos Estados Unidos e na Europa é bem conhecida, pois há vários livros sobre o assunto, dentre eles: Atlas of Skin Diseases of the Horse (Montes \& Vaughan 1983), Practical Equine Dermatology (Ackerman \& Pratt 1990), A Colour Atlas of Equine Dermatology (Pascoe 1990), Manual of Equine Dermatology (Pascoe \& Knottenbelt 1999), Equine Dermatology (Scott \& Miller Jr 2003), Practical Equine Dermatology (Lloyd et al. 2003) e Dermatology for the Equine Practitioner (Mueller 2005).

Estudos realizados no Reino Unido (Cotchin 1960), Austrália (Kerr \& Alden 1974), Kentucky (Bolin 1999a,b) e em várias regiões dos Estados Unidos (Ragland et al. 1970,
Scott \& Miller Jr. 2003, Sundberg et al. 1977) fornecem dados sobre a prevalência de tumores cutâneos em equinos e sugerem uma variação regional para a maior ou menor ocorrência de alguns. No entanto, muito pouca informação está disponível sobre a prevalência de dermatopatias em outras regiões geográficas do mundo, principalmente com relação a tumores não-neoplásicos e de lesões proliferativas semelhantes a tumor (Baker \& Leyland 1975, Scott \& Miller Jr 2003), e quase nada se sabe sobre a prevalência das dermatopatias em equinos no Brasil.

Dessa forma, a proposta desse estudo foi determinar a prevalência dos tumores cutâneos equinos diagnosticados no Laboratório de Patologia Veterinária (LPV) da Universidade Federal de Santa Maria (UFSM).

\section{MATERIAL E MÉTODOS}

Foram revisados os protocolos de biópsia de pele de equinos, arquivados no LPV-UFSM, entre janeiro de 1999 e dezembro de 2009 (11 anos). Dos protocolos de equinos que apresentavam tumores cutâneos, neoplásicos ou não e lesões inflamatórias semelhantes a tumor, foram coletadas informações referentes ao diagnóstico morfológico, ao sexo, à idade e à raça. Fizeram parte do estudo apenas os tumores que se originam da pele; tumores originados de qualquer mucosa foram excluídos. Em todos os casos, foram considerados os diagnósticos morfológicos que constavam nos protocolos. Os aspectos histológicos das lesões não foram revisados. Quanto ao sexo, os equinos foram classificados apenas como macho ou fêmea, independentemente de serem castrados. Quanto à idade, os equinos foram divididos em quatro categorias: menores de 1 ano, 1-5 anos, 6-14 anos e 15 anos ou mais; de acordo com critérios utilizados em um estudo (Pierezan et al. 2009). Foi calculada a prevalência total dos tumores.

\section{RESULTADOS E DISCUSSÃO}

De janeiro de 1999 a dezembro de 2009, foram recebidas no LPV 315 amostras de equinos. Dessas, 139 (44,1\%) eram amostras de pele de equinos com algum tipo de distúrbio dermatológico. Esse percentual é quase o dobro do encontrado em um estudo retrospectivo de 16 anos (19781994) realizado em um laboratório de diagnóstico da Universidade de Cornell que mostrou que $23,4 \%$ de todas as amostras de equinos recebidas eram de lesões cutâneas (Scott \& Miller Jr. 2003). Talvez essa diferença se deva ao período em que os trabalhos foram realizados, pois, nos últimos anos, parece ter ocorrido aumento no interesse dos clínicos e patologistas por dermatologia e dermatopatologia, respectivamente. É possível que esse maior interesse se deva ao fato de que as lesões de pele em equinos além de serem um problema estético, podem causar perdas econômicas (Scott \& Miller Jr 2003).

Das 139 amostras, 108 (77,6\%) eram de tumores cutâneos. Os tumores neoplásicos e as lesões inflamatórias e proliferativas não neoplásicas com aspecto tumoral (lesões tumoriformes) mais prevalentes foram: sarcoide (62/108 $[57,4 \%])$; carcinoma de células escamosas (11/108 $[10,2 \%]) ;$ pitiose (9/108 [8,3\%]); tecido de granulação (7/ 108 [6,5\%]; e granuloma eosinofílico $(4 / 108$ [3,8\%]). Os 
Quadro 1. Prevalência dos tumores cutâneos de equinos diagnosticados no Laboratório de Patologia Veterinária da Universidade Federal de Santa Maria (LPV-UFSM)

\begin{tabular}{lcc}
\hline \multicolumn{1}{c}{ Tumor } & N & $(\%)$ \\
\hline Sarcoide & 62 & 57,4 \\
Carcinoma de células escamosas & 11 & 10.2 \\
Pitiose & 9 & 8,3 \\
Tecido de granulação & 7 & 6,5 \\
Granuloma eosinofílico & 4 & 3,8 \\
Papiloma & 3 & 2,8 \\
Placa aural & 3 & 2,8 \\
Linfoma & 2 & 1,9 \\
Fibroma & 1 & 0,9 \\
Fibrossarcoma & 1 & 0,9 \\
Melanoma & 1 & 0,9 \\
Nevo de colágeno & 1 & 0,9 \\
Nevo linear & 1 & 0,9 \\
Pseudolinfoma & 1 & 0,9 \\
Calcinose circunscrita & 1 & 0,9 \\
Total & 108 & 100 \\
& &
\end{tabular}

outros tumores contaram com aproximadamente $14 \%$ dos casos (Quadro 1).

A prevalência do sarcoide neste estudo foi de $57,4 \%$; semelhante à descrita para a região noroeste dos Estados Unidos, que foi de 51,4\% (Valentine 2006), e para a região norte de Queesland, na Austrália, de 46,4\% (Miller \& Campbell 1982). No entanto, essa prevalência é bem maior que a descrita para o nordeste dos Estados Unidos, que foi de 35\% (Scott \& Miller 2003) e para o sudeste de Queensland, que representou $30 \%$ das lesões de pele de equinos (Pascoe \& Summers 1981). Esses resultados indicam, provavelmente, uma variação regional para a ocorrência desse tumor. De acordo com alguns autores (Scott \& Miller 2003), as raças de sela são mais predispostas ao desenvolvimento do sarcoide do que as raças de corrida. A raça Crioula foi a mais prevalente neste estudo (44/108 [40,7\%]) (Quadro 2); essa alta prevalência pode ser devido ao fato que no Rio Grande do Sul, cavalos dessa raça são os mais utilizados em esportes e no trabalho em fazendas (Pierezan et al. 2009). Quarenta e cinco por cento (28/62) dos cavalos com sarcoide pertenciam a essa raça. Esse resultado pode estar relacionado com o maior contato desses animais com o rebanho bovino e com consequente maior exposição ao papilomavírus (Chambers et al. 2003). Esse dado é semeIhante ao encontrado em outro estudo, em que raças de sela foram as duas mais afetadas (Valentine 2006).

Alguns autores (Rooney \& Robertson 1996, Scott \& Miller Jr 2003) descrevem que o sarcoide é comum em cavalos com menos de sete anos e raro naqueles com menos de um ano de idade. Esses dados são semelhantes aos que foram observados neste estudo em que trinta e dois cavalos $(51,6 \%)$ com sarcoide tinham entre $1-5$ anos de idade e 12 (19,4\%) tinham entre 6-14 anos (Quadro 3) e corroboram com outros (Marti et al. 1993, Ginn et al. 2007) que relatam que o sarcoide é mais observado em equinos entre três e seis anos.

Carcinoma de células escamosas foi o segundo tumor mais prevalente e representou $10,2 \%$ dos casos. Esse resultado assemelha-se ao encontrado na literatura internacional, a qual descreve o carcinoma de células escamosas como o segundo tumor mais importante nessa espécie, representando $6,9 \%$ a $37 \%$ dos tumores cutâneos (Scott \& Miller Jr. 2003). Esse resultado também é semelhante ao encontrado em outros estudos, em que o carcinoma de células escamosas representou 18,3\% (Valentine 2006) e 7,4\% (Miller \& Campbell 1982) dos casos. No entanto, num levantamento em que foram considerados todos os tipos de neoplasma,

Quadro 2. Prevalência dos tumores cutâneos de equinos diagnosticados no Laboratório de Patologia Veterinária da Universidade Federal de Santa Maria (LPV-UFSM) de acordo com a raça

\begin{tabular}{|c|c|c|c|c|c|c|c|c|c|c|c|}
\hline Tumor/raça & Crioula & $\mathrm{SRD}^{\mathrm{a}}$ & $\mathrm{QM}^{\mathrm{b}}$ & $\mathrm{PSI}$ & Pônei & PSC $^{d}$ & $\mathrm{ML}^{\mathrm{e}}$ & $\mathrm{BH}^{\mathrm{f}}$ & Percheron & N.I.g & Total \\
\hline Sarcoide & 28 & $\begin{array}{c}6 \\
(45,2 \%)\end{array}$ & $\begin{array}{c}6 \\
(9,7 \%)\end{array}$ & $\begin{array}{c}2 \\
(9,7 \%)\end{array}$ & $\begin{array}{c}1 \\
(3,2 \%)\end{array}$ & $\begin{array}{c}- \\
(1,6 \%)\end{array}$ & - & - & - & 19 & $\begin{array}{c}62 \\
(30,6 \%)\end{array}$ \\
\hline CCE $^{\mathrm{h}}$ & $\begin{array}{c}1 \\
(9,1 \%)\end{array}$ & $\begin{array}{c}2 \\
(18,2 \%)\end{array}$ & $\begin{array}{c}1 \\
(9,1 \%)\end{array}$ & $\begin{array}{c}1 \\
(9,1 \%)\end{array}$ & - & $\begin{array}{c}1 \\
(9,1 \%)\end{array}$ & $\begin{array}{c}1 \\
(9,1 \%)\end{array}$ & - & - & $\begin{array}{c}4 \\
(36,3 \%)\end{array}$ & 11 \\
\hline Pitiose & $\begin{array}{c}3 \\
(33,3 \%)\end{array}$ & $\begin{array}{c}2 \\
(22,2 \%)\end{array}$ & $\begin{array}{c}2 \\
(22,2 \%)\end{array}$ & - & - & - & - & - & - & $\begin{array}{c}2 \\
(22,2 \%)\end{array}$ & 9 \\
\hline T. granulaçãoi & $\begin{array}{c}2 \\
(28,5 \%)\end{array}$ & $\begin{array}{c}1 \\
(14,3 \%)\end{array}$ & - & $\begin{array}{c}1 \\
(14,3 \%)\end{array}$ & - & - & - & $\begin{array}{c}1 \\
(14,3 \%)\end{array}$ & $\begin{array}{c}1 \\
(14,3 \%)\end{array}$ & $\begin{array}{c}1 \\
(14,3 \%)\end{array}$ & 7 \\
\hline G. eosinof..j & $3(75 \%)$ & $1(25 \%)$ & - & - & - & - & - & - & - & - & 4 \\
\hline Papiloma & 2 & - & - & - & - & - & - & 1 & - & - & 3 \\
\hline Placa aural & 1 & - & - & - & - & 1 & - & 1 & - & - & 3 \\
\hline Linfoma & 1 & - & - & - & - & 1 & - & - & - & - & 2 \\
\hline Fibroma & - & 1 & - & - & - & - & - & - & - & - & 1 \\
\hline Fibrossarcoma & 1 & - & - & - & - & - & - & - & - & - & 1 \\
\hline Melanoma & - & - & - & - & - & - & - & - & - & 1 & 1 \\
\hline Nevo colágeno & 1 & - & - & - & - & - & - & - & - & - & 1 \\
\hline Nevo linear & 1 & - & - & - & - & - & - & - & - & - & 1 \\
\hline Pseudolinfoma & - & - & - & - & - & - & - & - & - & 1 & 1 \\
\hline C. circunsc. $^{\mathrm{k}}$ & - & - & - & - & - & - & - & - & - & 1 & 1 \\
\hline Total & $\begin{array}{c}44 \\
(40,7 \%)\end{array}$ & $\begin{array}{c}13 \\
(12,1 \%)\end{array}$ & $\begin{array}{c}9 \\
(8,3 \%)\end{array}$ & $\begin{array}{c}4 \\
(3,7 \%)\end{array}$ & $\begin{array}{c}1 \\
(0,9 \%)\end{array}$ & $\begin{array}{c}3 \\
(2,8 \%)\end{array}$ & $\begin{array}{c}1 \\
(0,9 \%)\end{array}$ & $\begin{array}{c}3 \\
(2,8 \%)\end{array}$ & $\begin{array}{c}1 \\
(0,9 \%)\end{array}$ & $\begin{array}{c}29 \\
(26,9 \%)\end{array}$ & 108 \\
\hline
\end{tabular}

a Sem raça definida; b Quarto-de-milha; ' Puro Sangue Inglês; d Puro Sangue de Corrida; e Mangalarga; ${ }^{\dagger}$ Brasileiro de Hipismo; 9 Não informado; ${ }^{\mathrm{h}}$ Carcinoma de células escamosas; i Tecido de granulação; j Granuloma eosinofílico; ${ }^{k}$ Calcinose circunscrita. 


\begin{abstract}
Quadro 3. Prevalência dos tumores cutâneos de equinos diagnosticados no Laboratório de Patologia Veterinária da Universidade Federal de Santa Maria (LPV-UFSM) de acordo com a faixa etária
\end{abstract}

\begin{tabular}{|c|c|c|c|c|c|c|}
\hline Tumor/faixa etária & $<1$ ano & 1-5 anos & 6-14 anos & $>15$ anos & N.I. ${ }^{a}$ & $\mathrm{~N}$ \\
\hline Sarcoide & - & $32(51,6 \%)$ & $12(19,4 \%)$ & $1(1,6 \%)$ & $17(27,4 \%)$ & 62 \\
\hline CCE $^{b}$ & - & - & $3(27,3 \%)$ & $6(54,5 \%)$ & $2(18,2 \%)$ & 11 \\
\hline Pitiose & $1(11,1 \%)$ & $2(22,2 \%)$ & $2(22,2 \%)$ & $3(33,4 \%)$ & $1(11,1 \%)$ & 9 \\
\hline Tecido de granulação & - & $2(28,6 \%)$ & $2(28,6 \%)$ & $1(14,3 \%)$ & $2(28,5 \%)$ & 7 \\
\hline Granuloma eosinofíl.c & - & $3(75 \%)$ & $1(25 \%)$ & - & - & 4 \\
\hline Papiloma & - & 3 & - & - & - & 3 \\
\hline Placa aural & - & 1 & - & - & 2 & 3 \\
\hline Linfoma & - & 1 & - & - & 1 & 2 \\
\hline Fibroma & - & - & - & 1 & - & 1 \\
\hline Fibrossarcoma & - & 1 & - & - & - & 1 \\
\hline Melanoma & - & - & 1 & - & - & 1 \\
\hline Nevo de colágeno & - & 1 & - & - & - & 1 \\
\hline Nevo linear & - & 1 & - & - & - & 1 \\
\hline Pseudolinfoma & - & - & - & - & 1 & 1 \\
\hline Calcinose circunscrita & - & - & - & - & 1 & 1 \\
\hline Total & $1(0,9 \%)$ & $47(43,5 \%)$ & $21(19,5 \%)$ & $12(11,1 \%)$ & $27(25 \%)$ & $108(100 \%)$ \\
\hline
\end{tabular}

a Não informado; b Carcinoma de células escamosas; ${ }^{c}$ Granuloma eosinofílico.

cutâneos ou não, e também os casos de necropsia, a prevalência desse tumor foi o dobro e representou $24,6 \%$ dos tumores de equinos (Sundberg 1977), o que ressalta a importância dessa condição na espécie em questão.

As três lesões não neoplásicas mais prevalentes eram inflamatórias e incluíram: pitiose (9/108 [8,3\%]), tecido de granulação exuberante (7/108 [6,5\%]) e granuloma eosinofílico $(4 / 108[3,8 \%])$. Em um levantamento realizado na Universidade de Oregon, em que foram avaliadas 116 lesões proliferativas não-neoplásicas, esses também foram os tumores mais prevalentes (Valentine 2005).

\section{CONCLUSÕES}

Com base nos achados deste estudo pode-se concluir que o sarcoide e o carcinoma de células escamosas são os tumores neoplásicos que mais comumente afetam a pele de equinos na região de abrangência do LPV-UFSM, e que a maioria das lesões tumorais não neoplásicas corresponde a casos de pitiose, tecido de granulação exuberante e granuloma eosinofílico.

\section{REFERÊNCIAS}

Ackerman L.J. \& Pratt P.W. 1990. Practical Equine Dermatology. American Veterinary Publications, Goleta. 256p.

Baker J.R. \& Leyland A. 1975. Histological survey of tumours of the horse, with particular reference to those of the skin. Vet. Rec. 96:419422.

Bolin D.C. 1999a. Equine sarcoid. Equine Dis. Quart. 7(3):6-8.

Bolin D.C. 1999b. Cutaneous neoplasms of horses. Equine Dis. Quart. 7(4):7-9.

Chambers G., Ellsmore V.A., O'Brien P.M., Reid S.W.J., Love S., Campo M.S. \& Nasir L. 2003. Association of bovine papillomavirus with the equine sarcoid. J. General Virol. 84:1055-1062.

Cotchin E. 1960. Tumours of farm animals: A survey of tumours examined at the Royal Veterinary College, London, during 1950-60. Vet. Rec. 72:816-823.

Cotchin E. 1977. A general survey of tumours in the horse. Eq. Vet. J. 9:16-21.

Ginn P.E., Mansell J.E.K.L. \& Rakich P.M. 2007. Skin and appendages, p.553-781. In: Maxie M.G. (Ed.), Jubb, Kennedy and Palmer's Pathology of Domestic Animals. Vol. $15^{\text {th }}$ ed. Elsevier, Philadelphia.

Kerr K.M. \& Alden C.L. 1974. Equine neoplasia-a ten year survey. Proc. Am. Assoc. Vet. Lab. Diagn. 17:183-187.

Lloyd D.H., Littlewood J.D., Craig J.M. \& Thomsett L.R. 2003. Practical Equine Dermatology. Blackwell Science Ltda, lowa, p.63-99.

Marti E., Lazary S., Antczak D.F. \& Gerber H. 1993. Report of the first international workshop on equine sarcoid. Equine Vet. J. 25:397-407.

Miller R.I. \& Campbell R.S. 1982. A survey of granulomatous and neoplastic diseases of equine skin in north Queensland. Aust. Vet. J. 1982 59(2):33-37.

Montes L.F. \& Vaughan J.T. 1983. Atlas of Skin Diseases of the Horse. W.B. Saunders, Philadelphia. 202p.

Mueller R.S. 2005. Dermatology for the Equine Practitioner. Ames, lowa. 97p.

Pascoe R.R. \& Knottenbelt D.C. 1999. Manual of Equine Dermatology. W.B Saunders, Philadelphia. 290p.

Pascoe R.R. \& Summers P.M. 1981. Clinical survey of tumours and tumour-like lesions in horses in south east Queensland. Equine Vet. J. 13(4):235-239.

Pascoe R.R. 1990. A Colour Atlas of Equine Dermatology. Wolfe, London. 142p.

Pierezan F., Rissi D.R., Rech R.R., Fighera R.A., Brum J.S. \& Barros C.S.L. 2009. Achados de necropsia relacionados com a morte de 335 equinos: 1968-2007. Pesq. Vet. Bras. 29:275-280.

Ragland W.L., Keown G.H. \& Spencer G.R. 1970. Equine sarcoid. Equine Vet. J. 2:2-11.

Scott D.W. \& Miller Jr W.H. 2003. Equine Dermatology. W.B. Saunders, St Louis, p.698-795.

Scott D.W. 2007. Color Atlas of Farm Animal Dermatology. Iowa: Blackwell. 252p.

Sundberg J.P., Burnstein T., Page E.H., Kirkham W.W. \& Robinson F.R. 1977. Neoplasms of Equidae. J. Am. Vet. Med. Assoc. 170:150-152.

Traub-Dargatz J.L., Salman M.D. \& Voss J.L. 1991. Medical problems of adult horses, as ranked by equine practitioners. J. Am. Vet. Med. Assoc. 198:1745-1747.

Valentine B.A. 2005. Equine cutaneous non-neoplastic nodular and proliferative lesions in the Pacific Northwest. Vet. Dermatol. 16(6):425428.

Valentine B.A. 2006. Survey of equine cutaneous neoplasia in the Pacific Northwest. J. Vet. Diagn. Invest. 18:123-126. 